\title{
Which Is the Optimal Method of Early Rehabilitation in Mechanically Ventilated Patients: a Protocol for Network Meta-analysis
}

\author{
Nannan Ding \\ Henan Provincial People's Hospital \\ Huizhen Peng \\ Henan Provincial People's Hospital \\ Yaru Guo \\ Henan Provincial People's Hospital \\ Haiyun Li \\ Henan Provincial People's Hospital \\ Wenli Zhao ( $\sim$ hhzhaowenli@126.com ) \\ Henan Provincial People's Hospital
}

\section{Protocol}

Keywords: early rehabilitation, mechanical ventilation, network meta-analysis, protocol

Posted Date: September 15th, 2021

DOI: https://doi.org/10.21203/rs.3.rs-856010/v1

License: (c) (i) This work is licensed under a Creative Commons Attribution 4.0 International License. Read Full License 


\section{Abstract}

Introduction Early rehabilitation of mechanically ventilated patients is safe, feasible and beneficial to prevent ICU-AW and its consequences. However, there are no guidelines or evidence-based studies about the optimal protocol of early rehabilitation. There is traditional meta-analysis about the effect of simple protocol, such as active mobilization, neuromuscular electrical stimulation, in-bed cycling exercise, interactive video games and so on, but no studies to explore the effects of multiple protocols together. For the extensive clinical implementation, it is important to conduct a network meta-analysis to compare the effect of different protocols of early rehabilitation.

Methods and analysis PubMed, Cochrane library, Web of Science, EMbase, grey literature and referent literature will be searched from inception to August 2019. Study selection, data extraction and quality assessment will be performed independently by two reviewers. The primary outcome is ICU acquired weakness (ICU-AW), secondary outcomes include the duration of mechanical ventilation and length of stay in ICU and hospital. Statistical analysis and graphical presentations will be conducted by R software (V.3.6.0). Cochrane Handbook for Systematic Reviews (V5.1.0) will be used to conduct the quality assessment. The rank possibility will be performed by the histogram. Statistical inconsistency assessment, sensitivity analysis and publication bias will be performed.

Discussion This protocol is prepared in accordance with the Preferred Reporting Items for Systematic Reviews and Meta-Analyses Protocols guidelines. The protocol gives an insight into the scope and parameters for the systematic review to be carried out.

\section{Registration number PROSPERO CRD42020141901}

\section{Introduction}

With the advancements of mechanical ventilation and techniques, critically ill patients' outcomes and survival rate have improved a lot over the last decades ${ }^{1-2}$. Studies showed that more than 5.7 million patients in the United States and more than 2 million Germany patients are admitted to intensive care units (ICUs) annually ${ }^{3}$; meanwhile, there were approximately $50 \%$ of ICU patients may experience muscle wasting and its consequences ${ }^{4}$. Mechanically ventilated patients will receive sedative and analgesic drugs to reduce distress and oxygen consumption; but on the other one hand, it will result in long periods of unconsciousness and immobility ${ }^{5}$, which will further increase muscle catabolism and decrease muscle protein synthesis and muscle mass ${ }^{6}$. The muscle dysfunction is called ICU-acquired weakness (ICU-AW), developing within the first week of ICU admission and was related to prolonged mechanical ventilation and high mortality ${ }^{6-8}$. Also, the consequences of it may persist for up to 5 years after hospital discharge, leading to physical dysfunction and low quality of life $\mathrm{e}^{9-10}$.

There were few effective therapies for preventing or treating ICU-AW and its' consequences ${ }^{11}$. However, some studies showed that early rehabilitation of mechanically ventilated patients is safe, feasible and 
beneficial to prevent ICU-AW and delirium, shorten the duration of mechanical ventilation, ICU and hospital stay, and reduce the time return back to normal life ${ }^{12-13}$. Studies indicated that active mobilisation reduce the mechanical ventilation duration, improve patients' muscle strength and the quality of life after hospital discharge $\mathrm{e}^{14-15}$. Which is the same as some evidence-based studies ${ }^{16-17}$. Other studies showed that neuromuscular electrical stimulation (NMES) has been used as an alternative to active exercise in some illness; it can improve peripheral microcirculation of critically ill patients after performing a forty-five minutes session of NMES on the lower extremities ${ }^{18-20}$. Also, some researchers showed that whole-body vibration (WBV) may have the potential to prevent and/or treat muscle weakness in critically ill patients ${ }^{21}$. There were no guideline or evidence-based studies to suggest selecting which protocol of early rehabilitation, thus hindering its extensive clinical implementation.

There were many traditional meta-analysis respectively studied the benefits of active mobilisation, NMES and so on, but can't compare multiple treatments simultaneously. Network meta-analysis (NMA) can compare all available treatments together even if there were no directed comparisons in one study and rank these interventions ${ }^{22}$. Hence, the purpose of our study is to evaluate which is the optimal protocol of early rehabilitation in mechanically ventilated patients through NMA.

\section{Methods And Analysis}

\section{Eligibility criteria}

\section{Types of study}

Randomized controlled trials (RCTs) will be included.

\section{Types of participants}

Patients aged $\geq 18$ years old with mechanical ventilation in ICU will be included.

\section{Types of intervention}

We are interested in any protocols of early rehabilitation to improve the outcomes of mechanically ventilated patients. Also, both simple intervention and multiple interventions will be included.

\section{Comparators}

We will make comparisons between usual care and early rehabilitation or between different protocols of early rehabilitation.

\section{Outcome measures}

\section{Primary outcome}

The incidence of ICU-AW of mechanically ventilated patients, which will be evaluated by MRC-score ${ }^{23}$. 


\section{Secondary outcomes}

We will include the duration of mechanical ventilation, length of stay in ICU and hospital.

\section{Search strategy}

PubMed, Cochrane library, Web of Science, EMbase, grey literature and referent literature will be searched from inception to August 2019. The search strategies will be ("early mobilization" OR "early rehabilitation" OR "active mobilization" OR "passive mobilization" OR "whole-body vibration" OR "early passive tilting" OR "early cognitive and physical rehabilitation" OR "passive leg cycling" OR "early In-Bed cycling" OR "inbed cycling exercise" OR "interactive video games" OR "electrical muscle stimulation" OR "neuromuscular electrical stimulation" OR "NMES") AND ("ICU" OR "intensive care unit*” OR "burn unit*” OR "coronary care unit*" OR "recovery room" OR "respiratory care unit*”). And an experienced expert will be invited to review the selected search strategy. In addition, we will manually search for the unpublished literature and will also try our best to contact the authors via email to require unreliable literature.

\section{Study selection}

All studies will be selected in the EndNote X8 which is a literature management software. Two authors will independently review the titles and abstracts of studies to screen studies, then review the full-text to include studies for quantitative analysis. A third reviewer will be invited for the discrepancies between the two authors.

\section{Data extraction}

Two authors will independently extract data according to a predesigned form, which will include study characteristics (first author, year, country, ICU type), patient characteristics (sample size, male/female, age, interventions, and controls), and outcomes (ICU-AW, duration of mechanical ventilation, and length of stay in ICU and hospital).

\section{Quality assessment}

Two reviewers will independently assess the risk of bias using the Cochrane Handbook for Systematic Reviews (V5.1.0), which includes random sequence generation, allocation concealment, participants/personnel blinding, outcome assessor blinding, incomplete outcome data, selective reporting, and other bias ${ }^{24}$. The third reviewer will be invited to solve the discrepancies of the two reviewers.

\section{Data synthesis and statistical methods}

We will use R software (V3.6.0, University of Auckland, New Zealand) to conduct statistical analysis and graphical presentations, which will included pairwise meta-analysis, ranking possibility, inconsistency analysis, subgroup analysis and sensitivity analyses; Stata software (V13.0, StataCorp, College Station, 
Texas, USA) will be used to assess publication bias by funnel plot, Begg or Egger's test if included studies were ten or more.

\section{Network meta-analysis}

Dichotomous outcomes will use odd ratio (OR) and $95 \%$ confidence interval $(95 \% \mathrm{Cl})$ as effect value; mean differences or standardised mean differences (SMDs) with $95 \% \mathrm{Cl}$ for continuous outcomes. Heterogeneity text will be assessed by using the $\chi^{2}$ test and $\mathrm{I}^{2}$ statistic. If $\mathrm{p}$ value $\geq 0.1$ and $\mathrm{I}^{2} \leq 50 \%$, we will consider there is no significant statistical heterogeneity exists and use the Mantel-Haenszel fixed effect model; if in contrast, we will use the random effects model. Sensitivity analysis will be conducted to explore the sources of heterogeneity. Publication bias or small sample effect will be examined through funnel plot, Begger or Egger's test if more than ten studies are available.

\section{Ranking possibility}

We will perform histogram of ranking probability to rank the intervention. The bigger of the value in the rank, the more possibility of it rank there.

\section{Assessment of inconsistency}

The consistency between direct and indirect evidence will be assessed by node split method in the loop, which separates direct evidence from indirect evidence for a specific comparison in the loop (inconsistency factor). $P$ value will be used to estimate the inconsistency, $p>0.05$ indicated it is consistency between direct and indirect comparisons, and it is inconsistency when $p \leq 0.05$.

\section{Subgroup analysis and sensitivity analysis}

Subgroup analyses and network meta-regression will be performed according to the ICU type, country and study quality when there is high heterogeneity. And we will perform sensitivity analyses by excluding each study to check the stability of the results.

\section{Publication bias}

Potential publication bias will be assessed by funnel plot, Egger or Begger's text.

\section{Quality of evidence}

The evidence quality will be assessed following the Grading of Recommendations, Assessment, Development and Evaluation (GRADE) method ${ }^{25}$.

\section{Limitations}

Firstly, there will be a inevitable limitation in this study that there is no librarian or information specialist included, which may result in literature searches are not complete and make bias ${ }^{26-27}$; Secondly, there 
may be potentially high heterogeneity because of the different interventions.

\section{Declarations}

\section{Ethics and dissemination}

Ethics approval is not required, because the present study is based on previous studies and no individual patients will be included in this review. This review will be published in a peer reviewed journal.

\section{Provenance and peer review}

Not commissioned; externally peer-reviewed.

\section{Authors' contributions}

$\mathrm{NN}, \mathrm{WL}$ and $\mathrm{HY}$ is responsible for the conception and design of this protocol. $\mathrm{NN}$ and $\mathrm{HZ}$ contributed to the data curation. Methodology were assessed by NN. NN drafted this protocol. NN and YR proofread the entire protocol. All authors read and approved the final manuscript.

\section{Funding}

The authors have not declared a specific grant for this research from any funding agency in the public, commercial or not-for-profit sectors.

\section{Consent for publication}

Not required.

\section{Competing interests}

None declared.

\section{References}

1. Van den Berghe G, Wilmer A, Hermans G, et al. Intensive insulin therapy in the medical ICU. N Engl J Med 2006;354:449-461.

2. Zambon $\mathrm{M}$, Vincent $\mathrm{JL}$. Mortality rates for patients with acute lung injury/ARDS have decreased over time. Chest 2008;133:1120-1127.

3. Farhan $\mathrm{H}$, Moreno-Duarte I, Latronico N, et al. Acquired muscle weakness in the surgical intensive care unit: nosology, epidemiology, diagnosis, and prevention. Anesthesiology 2016;124:207-234.

4. Zorowitz RD. ICU-acquired weakness. Chest 2016;150(4):966-971.

5. Schweickert WD, Pohlman MC, PohIman AS, et al. Early physical and occupational therapy in mechanically ventilated, critically ill patients: a randomised controlled trial. Lancet 2009;373:1874- 
1882.

6. Truong AD, Fan E, Brower RG, et al. Bench-to-bedside review: mobilizing patients in the intensive care unit-from pathophysiology to clinical trials. Crit Care 2009;13:216-223.

7. Dejonghe B, Sharshar T, Lefaucheur JP, et al. Paresis acquired in the intensive care unit: a prospective multicenter study. JAMA 2002;288:2859-2867.

8. Parry SM, El-Ansary D, Cartwright MS, et al. Ultrasonography in the intensive care setting can be used to detect changes in the quality and quantity of muscle and is related to muscle strength and function. Journal of Critical Care 2015;30(5):1151.e9-14.

9. Herridge MS, Tansey CM, MattéA, et al.Functional disability 5 years after acute respiratory distress syndrome.N Engl J Med 2011;364(14):1293-1304.

10. van der Schaaf M, Beelen A, Dongelmans DA, et al. Poor functional recovery after a critical illness: a longitudinal study. J Rehabil Med 2009;41:1041-1048.

11. Jones $C$, Skirrow P, Griffiths RD, et al. Rehabilitation after critical illness: A randomized, controlled trial. Crit Care Med 2003;31:2456-2461.

12. Schweickert WD, Pohlman MC, Pohlman AS, et al. Early physical and occupational therapy in mechanically ventilated, critically ill patients.Lancet 2009;373(9678):1874-1882.

13. Tipping CJ, Harrold M, Holland A, et al. The effects of active mobilisation and rehabilitation in ICU on mortality and function. Intensive Care Med 2017;43(2):171-183.

14. Hodgson CL, Stiller K, Needham DM, et al. Expert consensus and recommendations on safety criteria for active mobilization of mechanically ventilated critically ill adults. Crit Care 2014;18:1-9.

15. Schaller SJ, Anstey M, Blobner M, et al. Early, goal-directed mobilisation in the surgical Intensive Care Unit: a randomised controlled trial. Lancet 2016;388:1377-1388.

16. Stiller K. Physiotherapy in intensive care: an updated systematic review. Chest 2013;144:825-847.

17. Tipping CJ, Harrold M, Holland A, et al. The effects of active mobilisation and rehabilitation in ICU on mortality and function: a systematic review. Intensive Care Med 2017;43:171-183.

18. Nuhr MJ, Pette D, Berger R, et al. Beneficial effects of chronic low-frequency stimulation of thigh muscles in patients with advanced chronic heart failure. Eur Heart J 2004;25:136-143.

19. Vivodtzev I, Pépin JL, Vottero G, et al. Improvement in quadriceps strength and dyspnea in daily tasks after 1 month of electrical stimulation in severely deconditioned and malnourished COPD. Chest 2006;129:1540-1548.

20. Gerovasili V, Tripodaki E, Karatzanos E, et al. Short term systemic effect of electrical muscle stimulation in critically ill patients. Chest 2009;136:1249-1256.

21. Wollersheim $T$, Haas $K$, Wolf $S$, et al. Whole-body vibration to prevent intensive care unit-acquired weakness: safety, feasibility, and metabolic response. Critical Care 2017;21(1):9.

22. Rouse B, Chaimani A, Li T. Network meta-analysis: an introduction for clinicians. Intern Emerg Med 2017;12(1):103-111. 
23. Fan E, Cheek F, Chlan L, et al. An official American Thoracic Society Clinical Practice guideline: the diagnosis of intensive care unit-acquired weakness in adults. Am J Respir Crit Care Med 2014;190(12):1437-1446.

24. Higgins JP, Altman DG, Gøtzsche PC, et al. The Cochrane collaboration's tool for assessing risk of bias in randomised trials. BMJ 2011;343:d5928.

25. Puhan MA, Schünemann HJ, Murad MH, et al. A GRADE Working Group approach for rating the quality of treatment effect estimates from network meta-analysis. BMJ 2014;349:g5630.

26. Gao Y, Ge L, Ma X, et al.Improvement needed in the network geometry and inconsistency of Cochrane network meta-analyses: a cross-sectional survey. J Clin Epidemiol 2019;113:214-227.

27. Li L, Tian J, Tian H, et al. Network meta-analyses could be improved by searching more sources and by involving a librarian. J Clin Epidemiol 2014;67(9):1001-7.

\section{Supplementary Files}

This is a list of supplementary files associated with this preprint. Click to download.

- PRISMAchecklist.doc 